\title{
Microwave-gated dynamic nuclear polarization
}

Bornet, Aurélien; Pinon, Arthur; Jhajharia, Aditya; Baudin, Mathieu; Ji, Xiao; Emsley, Lyndon; Bodenhausen, Geoffrey; Ardenkjær-Larsen, Jan Henrik; Jannin, Sami

\section{Published in:}

Physical Chemistry Chemical Physics

Link to article, DOI:

$10.1039 / \mathrm{c} 6 \mathrm{cp} 05587 \mathrm{~g}$

Publication date:

2016

Document Version

Peer reviewed version

Link back to DTU Orbit

Citation (APA):

Bornet, A., Pinon, A., Jhajharia, A., Baudin, M., Ji, X., Emsley, L., Bodenhausen, G., Ardenkjær-Larsen, J. H., \& Jannin, S. (2016). Microwave-gated dynamic nuclear polarization. Physical Chemistry Chemical Physics, 18, 30530-30535. https://doi.org/10.1039/c6cp05587g

\section{General rights}

Copyright and moral rights for the publications made accessible in the public portal are retained by the authors and/or other copyright owners and it is a condition of accessing publications that users recognise and abide by the legal requirements associated with these rights.

- Users may download and print one copy of any publication from the public portal for the purpose of private study or research.

- You may not further distribute the material or use it for any profit-making activity or commercial gain

- You may freely distribute the URL identifying the publication in the public portal

If you believe that this document breaches copyright please contact us providing details, and we will remove access to the work immediately and investigate your claim 


\title{
Microwave Gated Dynamic Nuclear Polarization
}

\author{
Aurélien Bornet $^{\mathrm{a}}$, Arthur Pinon ${ }^{\mathrm{a}}$, Aditya Jhajharia ${ }^{\mathrm{b}, \mathrm{c}}$, Mathieu Baudin ${ }^{\mathrm{a}, \mathrm{b}, \mathrm{c}}$, Lyndon Emsley ${ }^{\mathrm{a}}$, \\ Geoffrey Bodenhausen ${ }^{\mathrm{a}, \mathrm{b}, \mathrm{c}}$, Jan Henrik Ardenkjaer-Larsen ${ }^{\mathrm{d}, \mathrm{e}}$ and Sami Jannin ${ }^{\mathrm{a}, \mathrm{f}, \mathrm{g} *}$
}

a Ecole Polytechnique Fédérale de Lausanne, Institut des Sciences et Ingénierie Chimiques, 1015 Lausanne, Switzerland.

b Département de Chimie, Ecole Normale Supérieure, PSL Research University, UPMC Univ Paris 06, CNRS, Laboratoire des Biomolécules (LBM), 24 rue Lhomond, 75005 Paris, France.

c Sorbonne Universités, UPMC Univ Paris 06, Ecole Normale Supérieure, CNRS, Laboratoire des Biomolécules (LBM), Paris, France.

${ }^{d}$ Department of Electrical Engineering, Technical University of Denmark, Lyngby 2800, Denmark.

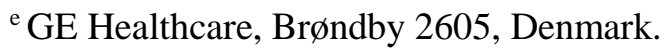

${ }^{f}$ Bruker BioSpin AG, Industriestrasse 26, 8117 Fällanden, Switzerland.

${ }^{g}$ Univ Lyon, CNRS, Université Claude Bernard Lyon 1, ENS de Lyon, Institut des Sciences Analytiques, UMR 5280, 5 rue de la Doua, 69100 Villeurbanne, France.

\section{Corresponding Author}

\footnotetext{
*Sami Jannin: sami.jannin@univ-lyon1.fr
} 
[Type here]

\section{Abstract}

Dissolution Dynamic Nuclear Polarization (D-DNP) has become a method of choice to enhance signals in Nuclear Magnetic Resonance (NMR). Recently, we have proposed to combine cross-polarization (CP) with D-DNP to provide high polarization $P\left({ }^{13} \mathrm{C}\right)$ in short build-up times. In this Letter, we show that switching the microwave irradiation off a few hundred milliseconds prior to CP can significantly boost the efficiency. By implementing microwave gating, ${ }^{13} \mathrm{C}$ polarizations on sodium $\left[1-{ }^{13} \mathrm{C}\right]$ acetate as high as $64 \%$ could be achieved with polarization build-up time constants as short as $160 \mathrm{~s}$. A polarization of $P\left({ }^{13} \mathrm{C}\right)=$ $78 \%$ could even be reached for $\left[{ }^{13} \mathrm{C}\right]$ urea.

\section{Key words}

Hyperpolarization, Dynamic Nuclear Polarization, Cross Polarization, Paramagnetic Relaxation, Microwave Gating.

\section{Abbreviations}

DNP: Dynamic Nuclear Polarization,

D-DNP: dissolution Dynamic Nuclear Polarization,

CP: Cross Polarization, TEMPOL: 4-hydroxy-2,2,6,6-tetramethylpiperidin-1-oxyl.

PA: paramagnetic agent

ESR: electron spin resonance 
[Type here]

\section{Introduction}

Dynamic Nuclear Polarization (DNP) [1, 2] has become a method of choice to enhance signals in Nuclear Magnetic Resonance (NMR). DNP usually consists in transferring electronic spin polarization to the surrounding nuclear spins via microwave irradiation in frozen glasses doped with paramagnetic polarizing agents (PAs) at suitable temperatures and magnetic fields. Enhancements in polarization $P\left({ }^{13} \mathrm{C}\right)$ in the liquid state at room temperature by factors exceeding 10,000 are possible using the so called dissolution-DNP (D-DNP) technique [3]. Here, the DNP sample is polarized as a frozen organic glass at low temperatures and moderate magnetic fields (typically $1.2<T<4.2 \mathrm{~K}$ and $B_{0}=6.7 \mathrm{~T}$ in our laboratories) where the electron spin polarization approaches unity, partly saturated by suitable microwave irradiation, dissolved and brought to room temperature with superheated water, and finally transferred to an NMR or MRI apparatus for detection. The method has found applications in the field of medical imaging, where hyperpolarized $\left[1-{ }^{13} \mathrm{C}\right]$ pyruvate or other metabolites can be used to monitor enzymatic conversion rates. This allowed real-time localized ${ }^{13} \mathrm{C}$ spectroscopy for tumour characterization in patients [4].

The D-DNP experiment was initially designed to polarize directly low-gamma nuclear spins, mainly ${ }^{13} \mathrm{C}$, using radicals such as Trityl that have narrow ESR lines. There are many advantages in polarizing ${ }^{13} \mathrm{C}$ nuclear spins as (i) they can be enriched, (ii) there is no significant background signal, (iii) and the longitudinal relaxation times can be as long as $T_{1}\left({ }^{13} \mathrm{C}\right)>50 \mathrm{~s}$ in carboxylic, carbonylic, or quaternary sites. This allows a significant part of the hyperpolarized magnetization of metabolites such as $\left[1-{ }^{13} \mathrm{C}\right]$ pyruvate to survive the transfer from the polarizer to the detection magnet, including infusion into animals or patients.

Recently, we have proposed to combine D-DNP with cross-polarisation (CP) [5-7]. The abundant proton spins are first rapidly polarized using PAs with broad ESR lines such as TEMPO. The proton polarization can be as high as $P\left({ }^{1} \mathrm{H}\right)>90 \%$ at $T=1.2 \mathrm{~K}$ and $B_{0}=6.7 \mathrm{~T}$. This can then be subsequently transferred to low-gamma spins such as ${ }^{13} \mathrm{C}$. This indirect strategy provides high polarizations $P\left({ }^{13} \mathrm{C}\right)$ in short build-up times.

In this Letter, we show that by gating the microwave irradiation off prior to $\mathrm{CP}$, the electron polarization returns to its highly polarized Boltzmann thermal equilibrium $P_{e}^{T E}$ on the time-scale of $T_{1}(\mathrm{e})$ which is on the order of $100 \mathrm{~ms}$ in our systems. As a result, the proton relaxation time in the rotating frame in presence of a radiofrequency field, $T_{1 p}\left({ }^{1} \mathrm{H}\right)$, can be extended by as much as an order of magnitude, while $T_{1 \rho}\left({ }^{13} \mathrm{C}\right)$ also increases, albeit by a smaller factor. This allows the CP contacts to be extended, thus significantly improving $\mathrm{CP}$ efficiency. By gating the microwaves, a polarization $P\left({ }^{13} \mathrm{C}\right)=$ $64 \%$ could be achieved in $\left[1-{ }^{13} \mathrm{C}\right]$ acetate with build-up time constants as short as $160 \mathrm{~s}$. A record 
[Type here]

polarization of $P\left({ }^{13} \mathrm{C}\right)=78 \%$ could be reached in $\left[{ }^{13} \mathrm{C}\right]$ urea, albeit with a somewhat longer build-up time constant of $470 \mathrm{~s}$. 


\section{[Type here]}

\section{Paramagnetic relaxation}

Under conditions that are suitable for DNP, a significant contribution to the relaxation rate constant in the rotating frame $1 / T_{1 \rho}\left({ }^{1} \mathrm{H}\right)$ can arise from the presence of PAs. This has been extensively studied in the presence and absence of spin diffusion [8,9], albeit only in the high temperature approximation. The spin-locked magnetization of a nuclear spin $I$ at a distance $r$ from an electron spin $S=$ $1 / 2$ relaxes with the following rate constant

$$
\frac{1}{T_{1 \rho}}=\frac{C}{r^{6}} \frac{\tau}{1+\omega_{1}^{2} \tau^{2}}
$$

with $r$ the distance between the electron and nuclear spin, $\omega_{1}$ the nuclear angular frequency in the rotating frame, and $\tau$ the rate of fluctuation of the electron dipolar field seen by the nuclear spins, and $\mathrm{C}$ a constant (see supplement for details on $\tau \quad \square \square \square \square$ C). This equation is however only valid in the high-temperature approximation where the electron spin polarization is negligible.

Microwave irradiation can shorten $\boldsymbol{T}_{\mathbf{1} \boldsymbol{\rho}}$. Typically, at low temperatures and high fields, the electron spin polarization in thermal equilibrium $P_{e}^{T E}$ can be close to unity, which leads to an attenuation of the transition rates within the electron spin manifold by a factor [10]

$$
\kappa=1-P_{e}^{\mu w} \cdot P_{e}^{T E}
$$

where $P_{e}^{\mu w}$ is the partly saturated electron spin polarization in the presence of microwave irradiation. This leads to an acceleration of the relaxation rate constant in the rotating frame:

$$
\frac{1}{T_{1 \rho}}=\frac{C}{r^{6}} \frac{\kappa \tau}{1+\omega_{1}^{2}(\kappa \tau)^{2}}
$$

Under magic angle spinning (MAS) conditions, typically $T=100 \mathrm{~K}$ and $B_{0}=9.4 \mathrm{~T}$, the electron spin polarization in thermal equilibrium is relatively low with $P_{e}^{T E}=6.3 \%$, which translates into $0.996<\kappa<$ 1 , so that $1 / T_{1 \rho}$ remains essentially the same with or without electron spin saturation by microwave irradiation. However, under D-DNP conditions, typically at $T=1.2 \mathrm{~K}$ and $B_{0}=6.7 \mathrm{~T}$, the electron spin polarization in thermal equilibrium is close to unity with $P_{e}^{T E}=99.89 \%$, which translates into 2.1 . $10^{-3}<\kappa<1$. Therefore one can expect an increase in $1 / T_{1 \rho}$ by orders of magnitude when microwave irradiation is applied to saturate the electron spin transition.

To verify this, $T_{1 \rho}\left({ }^{1} \mathrm{H}\right)$ relaxation curves of the proton spins were measured with a suitable pulse sequence (Fig. $1 \mathrm{a}$ ) at $B_{0}=9.4 \mathrm{~T}$ and $T=100 \mathrm{~K}$ (Fig. $1 \mathrm{~b}$ ) and $B_{0}=6.7 \mathrm{~T}$ and $T=1.2 \mathrm{~K}$ (Fig. $1 \mathrm{c}$ ) with and 


\section{[Type here]}

without microwaves at $263 \mathrm{GHz}$ and $188.3 \mathrm{GHz}$ respectively (in the latter case, we applied frequency modulation over a range $\Delta f_{\mu w}=50 \mathrm{MHz}$ at a rate $f_{\text {mod }}=10 \mathrm{kHz}$ [11]) in a frozen glassy sample containing $3 \mathrm{M}\left[1-{ }^{13} \mathrm{C}\right]$ acetate in $\mathrm{D}_{2} \mathrm{O}: \mathrm{H}_{2} \mathrm{O}$ :glycerol- $\mathrm{d}_{8}$ (v:v:v = 1:4:5) doped with $40 \mathrm{mM}$ TEMPOL as PA. As expected, the effect of microwave irradiation on $1 / T_{1 \rho}$ is insignificant at $B_{0}=9.4 \mathrm{~T}$ and $T=100$ $\mathrm{K}$, but is dramatic at $B_{0}=6.7 \mathrm{~T}$ and $T=1.2 \mathrm{~K}$.

a Spin-locking pulse sequence

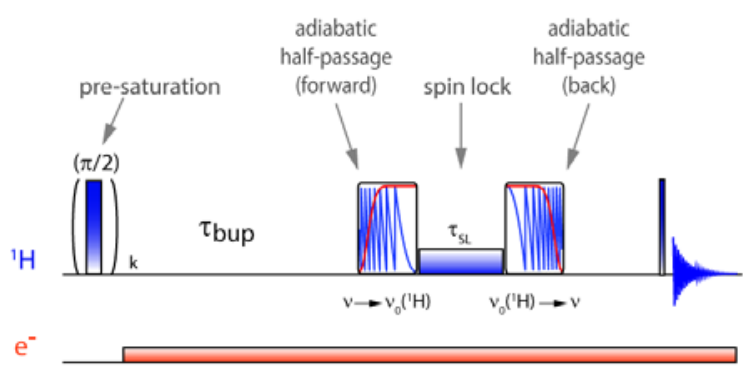

b $T_{1 p}$ with and without microwave irradiation at $100 \mathrm{~K}$

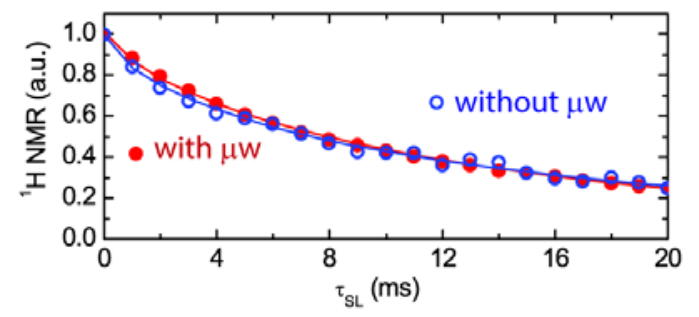

c $\mathrm{T}_{1 \rho}$ with and without microwave irradiation at $1.2 \mathrm{~K}$

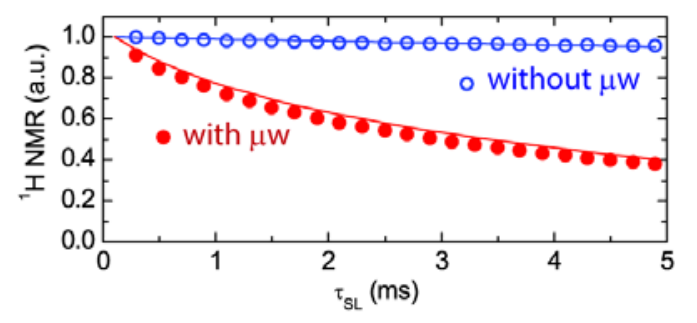

Figure 1: a) Pulse sequence used to measure $T_{1 \rho}\left({ }^{1} \mathrm{H}\right)$ where the proton spins are saturated by a train of $90^{\circ}$ pulses, and subsequently evolve during a fixed delay $\tau_{\text {bup }}=2 \mathrm{~s}$, either relaxing to their equilibrium if there is no microwave irradiation, or building up towards their DNP steady-state in the presence of microwave irradiation. After $\tau_{\text {bup }}$ has elapsed, a half-chirp pulse (swept from -100 kHz to the centre of the line in $175 \mu \mathrm{s}$, with a sweep rate of $0.57 \mathrm{kHz} / \mu \mathrm{s}$ and an amplitude $\left.\gamma B_{1} /(2 \pi)=20 \mathrm{kHz}\right)$ 
[Type here]

is applied to the protons to bring their magnetization to the transverse plane. This is followed by a spin-lock pulse with the same amplitude of $20 \mathrm{kHz}$ and a variable duration $\tau_{S L}$. Finally, the magnetization is flipped back along the longitudinal axis with a half-chirp pulse (swept from the centre of the line to $-100 \mathrm{kHz}$ in $175 \mu \mathrm{s}$, all other parameters being identical) and the proton NMR signal is observed following a $10^{\circ}$ excitation pulse. b) Proton $T_{1 \rho}$ decay measured at $B_{0}=9.4 \mathrm{~T}$ and $T$ $=100 \mathrm{~K}$ in a frozen glassy sample containing $3 \mathrm{M}\left[1-{ }^{13} \mathrm{C}\right]$ acetate in $\mathrm{D}_{2} \mathrm{O}: \mathrm{H}_{2} \mathrm{O}$ :glycerol- $\mathrm{d}_{8}$ (v:v:v = 1:4:5) doped with $40 \mathrm{mM}$ TEMPOL, with ( $\bullet$ ) and without (०) microwave irradiation with $P_{\mu w}=4 \mathrm{~W}$ and $f_{\mu w}=263 \mathrm{GHz}$. (the lines are drawn to guide the eye) c) Proton $T_{1 \rho}$ decay measured at $B_{0}=6.7$ $\mathrm{T}$ and $T=1.2 \mathrm{~K}$ in the same sample, with $(\bullet)$ and without $(\circ)$ microwave irradiation with $P_{\mu w}=87.5$ $\mathrm{mW}$ and $f_{\mu w}=188.3 \mathrm{GHz}$ modulated over a range $\Delta f_{\mu w}=50 \mathrm{MHz}$ at a rate $f_{\text {mod }}=10 \mathrm{kHz}$ (the lines are fitted to equation 4$)$.

Numerical simulations. Simulations of the relaxation curves were performed using Matlab ${ }^{\circledR}$. The model was implemented using a finite element routine for the description of the space and time domains, assuming a spherically symmetric system with one electron spin located at the centre, and surrounded by a spherical medium or radius c containing nuclear spins. Nuclear spins closer than $10 \AA$ to the electron were considered to be inside a "diffusion barrier" across which no spin diffusion can occur. Nuclear spins in this diffusion barrier are subjected to strong dipolar couplings to electrons leading to large splittings or frequency shifts; they are therefore considered as 'NMR invisible' and consequently simply neglected[12]. Such a system can be described by a partial differential equation [8].

$$
\frac{\partial P(r, t)}{\partial t}=\frac{1}{T_{1 \rho}} P(r, t)+\beta P(r, t)+D \nabla^{2} P(r, t)
$$

where $P$ is the proton polarization, $D$ is the diffusion constant, and $\beta$ is a leakage parameter that describes all sources of relaxation other than paramagnetic effects due to the unpaired electrons of TEMPOL. The initial polarization at time $t=0$ can be arbitrarily set to $P(r, 0)=1$ and the boundary conditions at the edges of the system can be set to $\partial P(r, t) / \partial r=0$. The equation was integrated numerically over space using trapezoidal steps, yielding the total NMR signal intensity as a function of time. Experimental relaxation curves were fitted using the least-squares method. Best fits were obtained with fixed parameters for the diffusion barrier $b=10 \AA$ (see [13] for a discussion), the diffusion constant $D=10 \mathrm{~nm}^{2} / \mathrm{s}$, and the non-paramagnetic leakage relaxation rate $\beta=-8.6 \mathrm{~s}^{-1}$ and with free parameters that converged robustly to a sphere radius $c=2 \mathrm{~nm}, C=2.3 \times 10^{-8} \mu \mathrm{m}^{6} / \mathrm{s}^{2}$, and $\tau=2,7690.10^{-4}$ (see supplement for the numerical simulations convergence). In the absence of microwave irradiation, the thermal equilibrium polarization 


\section{[Type here]}

$P_{e}^{T E}$ depends only on the sample temperature, i.e., $P_{e}^{T E}=99.89 \%$ at $1.2 \mathrm{~K}$. In the presence of microwave irradiation, the electron polarization $P_{e}^{\mu w}$ was treated as an adjustable temperature-dependent parameter and was estimated to be $P_{e}^{\mu w}=48 \%$ at $1.2 \mathrm{~K}$, well above $P_{e}^{\mu w}=0 \%$ that would describe complete saturation. Even a partial saturation of the electron spin polarization of $P_{e}^{\mu w}=48 \%$ severely affects the proton relaxation rate constant in the rotating frame $1 / T_{1 \rho}\left({ }^{1} \mathrm{H}\right)$. This in turn determines how much magnetization remains after spin-locking during an interval $\tau_{\mathrm{SL}}$. When the microwaves are switched off, we determined $T_{1 \rho}\left({ }^{1} \mathrm{H}\right) \approx 200 \mathrm{~ms}$, so that more than $90 \%$ of the spin-locked proton magnetization survives after spin-locking for $\tau_{\mathrm{SL}}=10 \mathrm{~ms}$, but this value drops to $20 \%$ when the microwaves are switched on, since $T_{1 \rho}\left({ }^{1} \mathrm{H}\right) \approx 10 \mathrm{~ms}$ in this case.

Fitting the $T_{1 \rho}$ relaxation curves with our method offers a way to estimate the electron spin polarization $P_{e}^{\mu w}$. Figure 2a shows seven $T_{1 \rho}$ relaxation curves measured with the sequence of Fig. 1a with different microwave powers $P_{\mu w}$ ranging from 6.25 to $87 \mathrm{~mW}$, and fitted to Eq. 4. The only parameter that varies between these experiments is the extent of saturation of the electron spin resonance, and hence the electron-spin polarization under microwave irradiation $P_{e}^{\mu w}$. The electron spin polarizations $P_{e}^{\mu w}$ deduced from the fits are reported in Fig. $2 \mathrm{~b}$ as a function of the applied microwave power $P_{\mu w}$.

a $\mathrm{T}_{10}$ curves varying microwave power

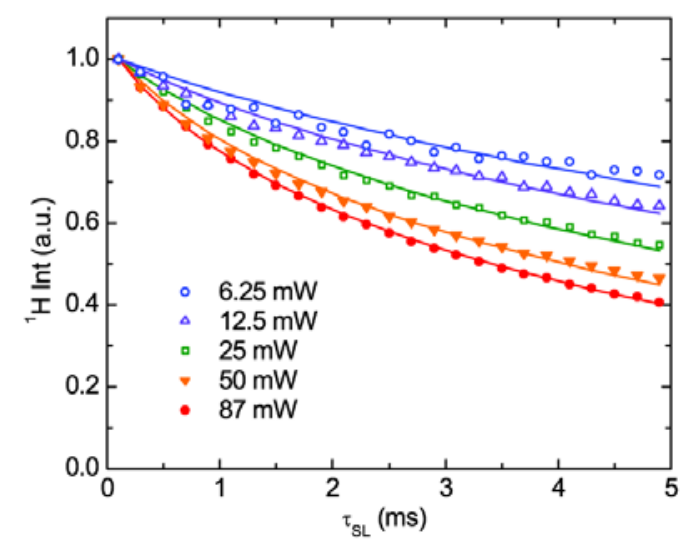

b Average electron spin polarization under microwave irradiation

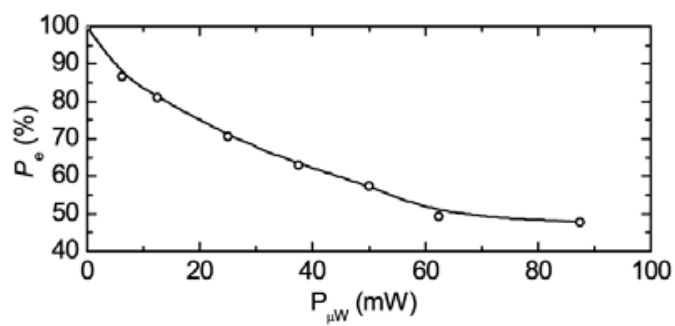




\section{[Type here]}

Figure 2: a) $T_{1 \rho}\left({ }^{1} \mathrm{H}\right)$ decay of the proton magnetization measured with the sequence of Fig. 1a as a function of the applied microwave power $P_{\mu w}$ (with $f_{\mu w}=188.3 \mathrm{GHz}, \Delta f_{\mu w}=50 \mathrm{MHz}, f_{\text {mod }}=10$ $\mathrm{kHz}$ ) in $3 \mathrm{M}\left[1-{ }^{13} \mathrm{C}\right]$ acetate in $\mathrm{D}_{2} \mathrm{O}: \mathrm{H}_{2} \mathrm{O}$ :glycerol- $\mathrm{d}_{8}$ (v:v:v = 1:4:5) doped with $40 \mathrm{mM}$ TEMPOL, at $1.2 \mathrm{~K}$ and $6.7 \mathrm{~T}$ (the lines are fitted to Eq. 4 with $P_{e}^{\mu w}$ as the only free parameter). This behaviour reflects varying degrees of saturation of the electron polarization. b) Average electron spin polarisations under microwave irradiation $P_{e}^{\mu w}$ (averaged over the whole ESR line) as a function of the power $P_{\mu w}$ of the microwave irradiation, estimated by numerical fits to Eq. 4 (the line is drawn to guide the eye).

Microwave gating extends $\boldsymbol{T}_{\mathbf{1} \boldsymbol{\rho}}$. Microwave irradiation is essential to DNP experiments but as seen above the partial saturation of the electron spins concurrently leads to shortening nuclear $T_{1 \rho}$. We propose here to interrupt the microwave irradiation during a time $\tau_{\text {gate }} \sim 5 T_{1 e}$ prior to spin-locking, in order to allow the electrons to relax to their highly polarized thermal equilibrium $P_{e}^{T E}$ at $1.2 \mathrm{~K}$ and $6.7 \mathrm{~T}$. Fig. 3a shows the spin-locking sequence with microwave gating (the pulse parameters for the protons are the same as in Fig. 1a). Fig. 3b displays the proton signal integral measured after spin locking $\left(\gamma B_{1}\left({ }^{1} \mathrm{H}\right) /(2 \pi)=20 \mathrm{kHz}\right.$, $\left.\tau_{S L}=5 \mathrm{~ms}\right)$ as a function of the microwave gating interval $\tau_{\text {gate }}$. Clearly, the relaxation time $T_{1 \rho}\left({ }^{1} \mathrm{H}\right)$ is extended as the electron spin polarization relaxes back to its highly polarized state after switching off the microwave irradiation. As a result, the proton signal that survives the spin locking experiment increases when the gating period is extended, until it reaches a plateau. The electron spin polarization was simulated using Eq. 4 as function of $\tau_{\text {gate }}$ with $t=5 \mathrm{~ms}, b=10 \AA, c=2 \mathrm{~nm}, D=10 \mathrm{~nm}^{2} / \mathrm{s}, C=-0.4790 \mu \mathrm{m}^{6} / \mathrm{s}^{2}, \tau=$ $2,7690.10^{-4} \mathrm{~s}$ and $\beta=-8.6047 \mathrm{~s}^{-1}$ (Fig. 3c). Comparison between experiments and simulations allowed us to estimate the electron spin-lattice relaxation time $T_{1 e}=48 \pm 1 \mathrm{~ms}$. We systematically used a microwave gating interval $\tau_{\text {gate }}=500 \mathrm{~ms}\left(\gg T_{1 e}\right)$ in all subsequent experiments. 
[Type here]

a

Gated spin-locking pulse sequence

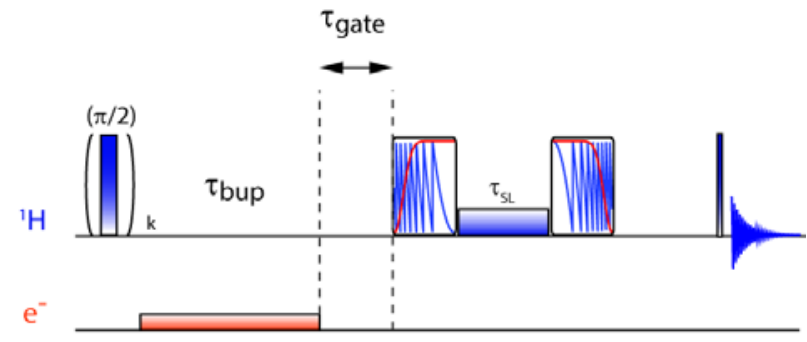

b 'H signal varying $\tau_{\text {gate }}$

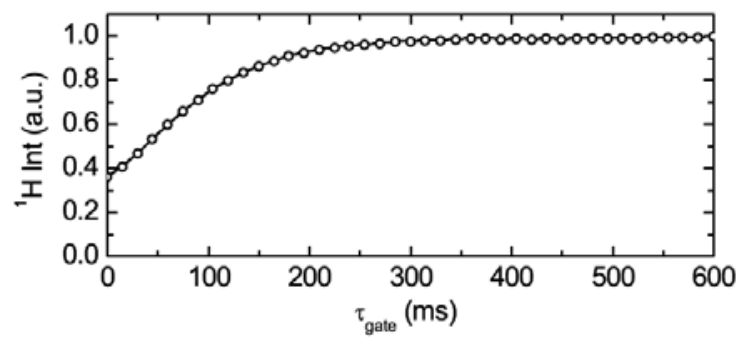

c Estimated electron spin polarization varying $\tau_{\text {gate }}$

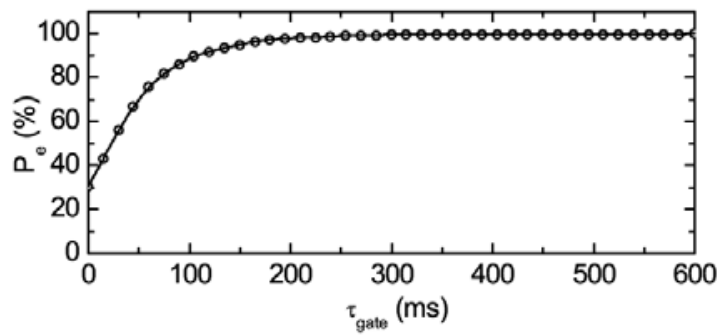

Figure 3: a) Pulse sequence used to determine $T_{1 \rho}\left({ }^{1} \mathrm{H}\right)$ with microwave gating. b) Proton signal integrals in $3 \mathrm{M}\left[1{ }^{13} \mathrm{C}\right]$ acetate in $\mathrm{D}_{2} \mathrm{O}: \mathrm{H}_{2} \mathrm{O}$ :glycerol- $\mathrm{d}_{8}$ (v:v:v = 1:4:5) doped with $40 \mathrm{mM}$ TEMPOL measured at $B_{0}=6.7 \mathrm{~T}$ and $T=1.2 \mathrm{~K}$ after spin locking $\left(\gamma B_{1}=20 \mathrm{kHz}, \tau_{S L}=5 \mathrm{~ms}\right)$ as a function of the gating interval $\tau_{\text {gate }}$ with the microwave parameters $P_{\mu w}=87.5 \mathrm{~mW}, f_{\mu w}=188.3 \mathrm{GHz}, \Delta f_{\mu w}=$ $50 \mathrm{MHz}$, and $f_{\text {mod }}=10 \mathrm{kHz}$, (a line was drawn to guide the eye). c) Simulated recovery of the electron spin polarization $P_{e}$ in the range $P_{e}{ }^{\mu W}<P_{e}<P_{e}{ }^{T E}$ as a function of the interval $\tau_{\text {gate }}$ by fitting $T_{1 e}$ to match the experimental data in $\mathbf{b}$; the a mono-exponential curve has a characteristic time constant $T_{1 e}=48 \mathrm{~ms}$.

The advantages of microwave gating are substantial at low temperatures. Fig. 4 shows the $T_{1 \rho}$ relaxation curves measured for different temperatures with the pulse sequence of Fig. 3a, with continuous or gated microwaves (fixed gating interval $\tau_{\text {gate }}=500 \mathrm{~ms}$ and $\tau_{S L}$ varied from 0 to $10 \mathrm{~ms}$ ). With continuous microwaves, all $T_{1 \rho}$ curves are alike, featuring a fast decay of the magnetization during spin 


\section{[Type here]}

locking. However, with gated microwaves, the magnetization can survive spin locking remarkably well, and especially at the lowest temperature $T=1.2 \mathrm{~K}$ where $\kappa=0.00055$. On the other hand, the advantages of microwave gating diminish at higher temperatures as the thermal equilibrium electron spin polarization $P_{e}^{T E}$ becomes significantly lower that unity.

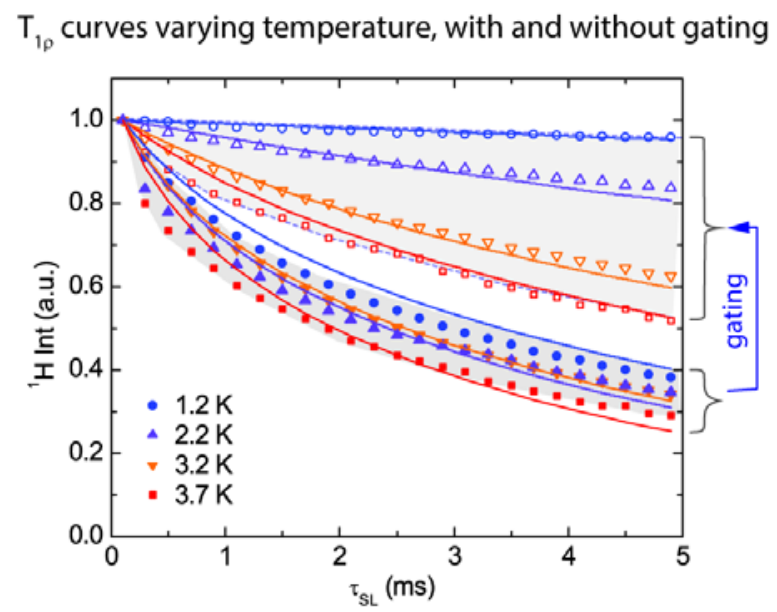

Figure 4: $T_{1 \rho}\left({ }^{1} \mathrm{H}\right)$ decay of the proton magnetization in $3 \mathrm{M}\left[1-{ }^{13} \mathrm{C}\right]$ acetate in $\mathrm{D}_{2} \mathrm{O}: \mathrm{H}_{2} \mathrm{O}$ :glycerol- $\mathrm{d}_{8}$ (v:v:v $=1: 4: 5)$ doped with $40 \mathrm{mM}$ TEMPOL with continuous or gated microwaves $\left(P_{\mu w}=87.5 \mathrm{~mW}\right.$, $f_{\mu w}=188.3 \mathrm{GHz}, \Delta f_{\mu w}=50 \mathrm{MHz}, f_{\text {mod }}=10 \mathrm{kHz}$ ) as a function of the sample temperature, i.e., as function of the electron Boltzmann polarization $P_{e}^{T E}$. The fast decays observed with continuous microwaves (lower curves) reflect varying degrees of saturation of the electron polarization, while the behaviour with gated microwaves (upper curves) reflects the thermal electron spin polarization $P_{e}{ }^{T E}$ that depends on the sample temperature (lines are numerical fits to Eq. 4).

Microwave gating improves the efficiency of cross polarization. We have recently shown that D-DNP can be boosted by performing ${ }^{1} \mathrm{H} \rightarrow{ }^{13} \mathrm{C}$ cross-polarization (CP) in combination with DNP [14-18]. Here we show that the microwave gating strategy can greatly improve the CP efficiency. Fig. 5a shows the pulse sequence used for CP with gated microwaves which is simply an extension of the sequence of Fig. 3a with spin locking also applied on ${ }^{13} \mathrm{C}$ to allow Hartmann-Hahn contact between ${ }^{1} \mathrm{H}$ and ${ }^{13} \mathrm{C}$ (pulse parameters for ${ }^{1} \mathrm{H}$ and ${ }^{13} \mathrm{C}$ are the same as in Fig. 1a, except that a $50 \% \rightarrow 100 \%$ ramp with an average $\gamma B_{1}\left({ }^{1} \mathrm{H}\right) /(2 \pi)=$ $20 \mathrm{kHz}$ rather than a rectangular excitation is applied to ${ }^{13} \mathrm{C}$ during $\tau_{C P}$ ). Fig. 5b shows the ${ }^{13} \mathrm{C}$ magnetization as a function of the $\mathrm{CP}$ contact time $\tau_{C P}$, without and with microwave gating. With continuous microwaves, the optimum CP contact time lies around $\tau_{C P}=1.5 \mathrm{~ms}$ before the decay of the ${ }^{1} \mathrm{H}$ magnetization (see Fig. 4a) compromises the transfer of polarization to ${ }^{13} \mathrm{C}$. With gated microwaves, CP contact times can be much longer (in this example with an optimum around $\tau_{C P}=20 \mathrm{~ms}$ ) thanks to the extended relaxation time $T_{1 \rho}\left({ }^{1} \mathrm{H}\right)$ (see Fig. 4), leading to a significantly improved CP efficiency (more 


\section{[Type here]}

than 50\% improvement in our sample). Note that this improvement is expected to be even more important for systems where the magnetization is transferred to ${ }^{13} \mathrm{C}$ from remote protons and necessitates extended CP contact times (for example for deuterated molecules immersed in a protonated solvent [18]).

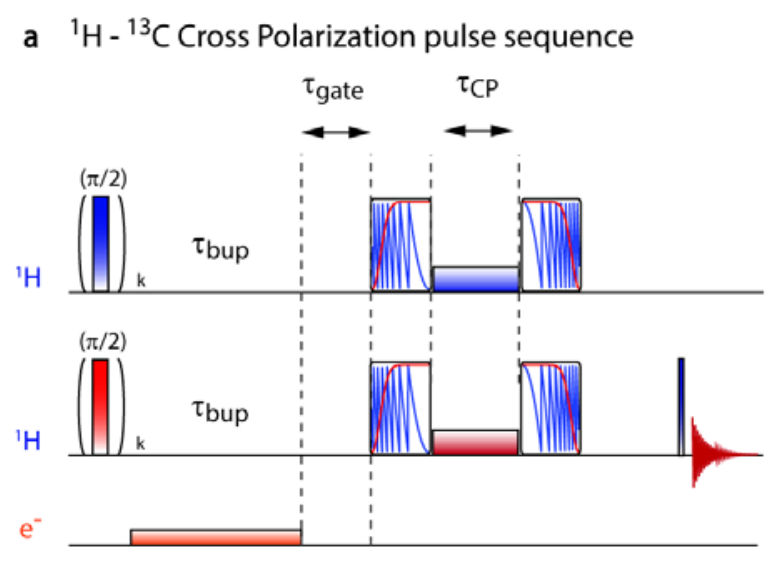

b CP efficiency varying $\tau_{\mathrm{CP}}$ with gated or continuous microwaves.

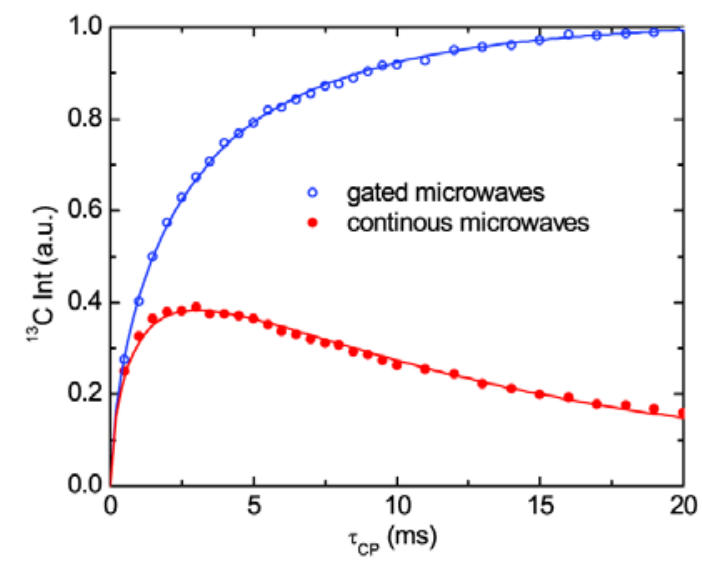

Figure 5: a) Pulse sequence for multiple-contact cross-polarization enhanced by DNP. The adiabatic half passage pulses sweep over $100 \mathrm{kHz}$ in $175 \mu \mathrm{s}$. The rf amplitude for ${ }^{13} \mathrm{C}$ is ramped from $50 \%$ to $100 \%$ of the average rf amplitude which was $\gamma B_{1}\left({ }^{1} \mathrm{H}\right) /(2 \pi)=20 \mathrm{kHz}$ on both channels. The microwave irradiation is gated off during the intervals $\tau_{\text {gate }}=500 \mathrm{~ms}$ and $\tau_{\mathrm{CP}}$. b) Signal amplitude of ${ }^{13} \mathrm{C}$ after a single CP transfer from ${ }^{1} \mathrm{H}$ to ${ }^{13} \mathrm{C}$ as a function of the $\mathrm{CP}$ contact time $\tau_{\mathrm{CP}}$, with gated $(\mathrm{\circ})$ or continuous $(\bullet)$ microwave irradiation $\left(P_{\mu w}=87.5 \mathrm{~mW}, f_{\mu w}=188.3 \mathrm{GHz}, \Delta f_{\mu w}=50 \mathrm{MHz}, f_{\text {mod }}=\right.$ $10 \mathrm{kHz}$ ) in $3 \mathrm{M}$ sodium $\left[1-{ }^{13} \mathrm{C}\right]$ acetate with $40 \mathrm{mM}$ TEMPOL at $1.2 \mathrm{~K}$ and $6.7 \mathrm{~T}$. Details of the fits are given in the supplement..

In a D-DNP experiment, one aims at building up the highest possible ${ }^{13} \mathrm{C}$ polarization prior to dissolution. We have shown that the ${ }^{13} \mathrm{C}$ polarization can be maximized by applying multiple $\mathrm{CP}$ contacts at intervals 


\section{[Type here]}

$\Delta_{C P} \sim \tau_{D N P}\left({ }^{1} H\right)$. The time interval $\Delta_{C P}$ allows for the proton polarization to be replenished by DNP before being drained again during the next CP contact. The use of microwave gating in this context has two favourable outcomes; (i) reducing losses of proton magnetization during spin locking (as shown in Figure 4) and (ii) improving the CP transfer efficiency (shown in Fig. 5). Altogether, the efficiency of the multiple CP sequence is greatly improved by microwave gating, as shown in Fig. 6 where the evolution of both ${ }^{1} \mathrm{H}$ and ${ }^{13} \mathrm{C}$ polarizations is followed using small nutation angle pulses in the course of a multiple $\mathrm{CP}$ experiment. With a fixed interval $\Delta_{C P}=160 \mathrm{~s}$, we applied optimal CP contact durations $\tau_{C P}=16 \mathrm{~ms}$ and $\tau_{C P}=1.5 \mathrm{~ms}$ respectively, which led to $P\left({ }^{13} \mathrm{C}\right)=64 \%$ with effective build-up time constants $\tau_{D N P}{ }^{C P}\left({ }^{13} C\right)=168 \mathrm{~s}$ with microwave gating. This compares favorably with and $P\left({ }^{13} \mathrm{C}\right)=38 \%$ with $\tau_{D N P}{ }^{C P}\left({ }^{13} C\right)=190 \mathrm{~s}$ with continuous microwaves. Using microwave gating thus enhances the build-up of the final ${ }^{13} \mathrm{C}$ polarization by a factor 1.7 .

a ${ }^{13} \mathrm{C} C P$ build-ups (gated and $\mathrm{cw}-\mu \mathrm{w} \mathrm{CP}$ )

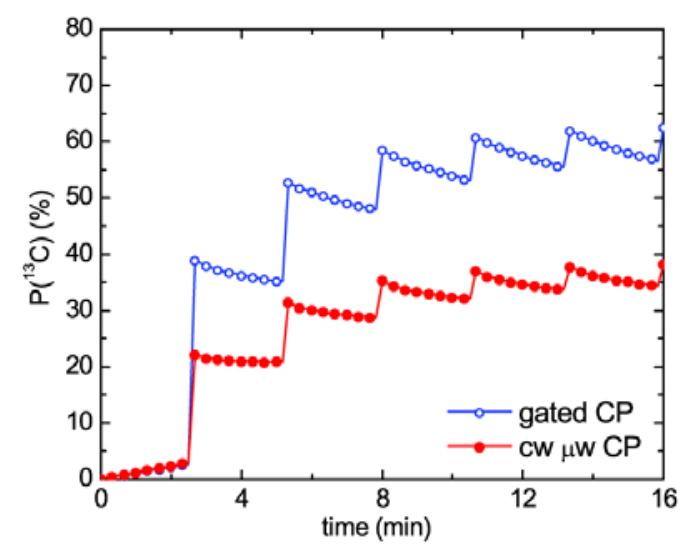

b ${ }^{1} \mathrm{H}$ build-ups (free, or during gated and $\mathrm{CW}-\mu \mathrm{w} \mathrm{CP}$ )

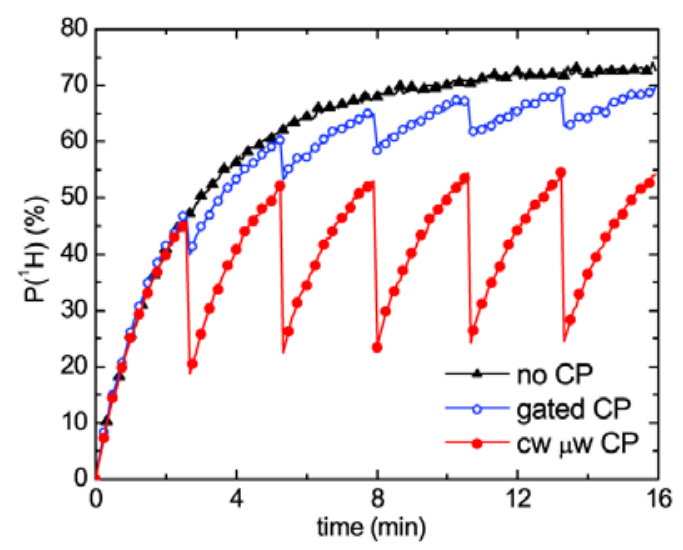

Figure 6: (a) Build-up of polarization $P\left({ }^{13} \mathrm{C}\right)$ during a multiple $\mathrm{CP}$ pulse sequence (see Fig. 5a) applied every 2.5 minutes with continuous $(\bullet)$ or gated $(\circ)$ microwave irradiation $\left(P_{\mu w}=87.5 \mathrm{~mW}\right.$, $\left.f_{\mu w}=188.3 \mathrm{GHz}, \Delta f_{\mu w}=50 \mathrm{MHz}, f_{\text {mod }}=10 \mathrm{kHz}\right)$ in $3 \mathrm{M}$ sodium $\left[1-{ }^{13} \mathrm{C}\right]$ acetate with $40 \mathrm{mM}$ TEMPOL at $1.2 \mathrm{~K}$ and $6.7 \mathrm{~T}$. (b) DNP build-up of proton polarization $P\left({ }^{1} \mathrm{H}\right)$ in the same sample and conditions without any $\mathrm{CP}(\mathbf{\Delta})$ or during multiple $\mathrm{CP}$ applied every 2.5 minutes with continuous microwave irradiation $(\bullet)$ or during multiple CP with gated microwave irradiation (०) (all lines are drawn to guide the eye).

As the losses of $P\left({ }^{1} \mathrm{H}\right)$ during $\mathrm{CP}$ contacts are greatly reduced by microwave gating, the proton polarization in the course of a CP-DNP sequence with gated microwaves almost reaches the same value as when CP is not used. In samples containing methyl groups, such as sodium pyruvate or sodium acetate, 


\section{[Type here]}

the steady state is typically limited to $P\left({ }^{1} \mathrm{H}\right)=70 \%$ because of relaxation induced by the rotation of the $\mathrm{CH}_{3}$ groups. Using non-methylated DNP samples, such as $\left[{ }^{13} \mathrm{C}\right]$ urea dissolved in $\mathrm{H}_{2} \mathrm{O}: \mathrm{D}_{2} \mathrm{O}$ :glycerol- $\mathrm{d}_{8}$ (v:v:v = 1:4:5) with $40 \mathrm{mM}$ TEMPOL, $P\left({ }^{1} \mathrm{H}\right)$ builds up to $90 \%$ under our DNP conditions at $1.2 \mathrm{~K}$. Using CP with microwave gating, it was possible to reach a polarization of $P\left({ }^{13} \mathrm{C}\right)=78 \%$ for this sample.

\section{Conclusions}

Low temperature DNP relies on the steady-state saturation of the electron spins of paramagnetic polarizing agents through microwave irradiation. We have shown in this Letter how this saturation leads to a significant deleterious shortening of the nuclear spin relaxation times in the rotating frame $T_{1 \rho}\left({ }^{1} \mathrm{H}\right)$. This is supported by numerical simulations with a simple paramagnetic relaxation model. The shortening of $T_{1 \rho}$ is obviously detrimental for cross-polarisation from ${ }^{1} \mathrm{H}$ to ${ }^{13} \mathrm{C}$ or other low-gamma nuclei. However, we demonstrate that this can be avoided by briefly gating off the microwave irradiation prior to cross-polarisation., For ${ }^{1} \mathrm{H} \rightarrow{ }^{13} \mathrm{C}$ cross-polarization, the final ${ }^{13} \mathrm{C}$ polarization can be boosted by $70 \%$ (i.e., increased by a factor 1.7$)$, resulting in $P\left({ }^{13} \mathrm{C}\right)=64 \%$ in $\left[1-{ }^{13} \mathrm{C}\right]$ acetate with a build-up time constant of 160 s, and $P\left({ }^{13} \mathrm{C}\right)=78 \%$ in $\left[{ }^{13} \mathrm{C}\right]$ urea with at time constant of $470 \mathrm{~s}$. The characteristic time constant of the return of the electron spins to their thermal equilibrium can be determined by simulations and was found to be $T_{1 e}=70 \mathrm{~ms}$ for the system under investigation at $1.2 \mathrm{~K}$.

\section{Acknowledgements}

The authors thank Anto Barisic and Dr Pascal Miéville from EPFL for valuable assistance, and Prof. Moreno Lelli for fruitful discussions. This work was supported by the Swiss National Science Foundation (SNSF), the Ecole Polytechnique Fédérale de Lausanne (EPFL), Bruker BioSpin Switzerland, the CNRS, and the European Research Council (ERC), Grant Agreement 339754 “Dilute para-water”.

\section{References}

[1] Carver, T.R. and C.P. Slichter, Polarization of Nuclear Spins in Metals, Phys. Rev. 92 (1953) 212213.

[2] Overhauser, A.W., Polarization of Nuclei in Metals, Phys. Rev. 92 (1953) 411-415. 
[Type here]

[3] Ardenkjaer-Larsen, J.H., B. Fridlund, A. Gram, G. Hansson, L. Hansson, M.H. Lerche, R. Servin, M. Thaning, and K. Golman, Increase in signal-to-noise ratio of $>10,000$ times in liquid-state NMR, Proc. Natl. Acad. Sci. USA 100 (2003) 10158-10163.

[4] Nelson, S.J., J. Kurhanewicz, D.B. Vigneron, P.E.Z. Larson, A.L. Harzstark, M. Ferrone, M. van Criekinge, J.W. Chang, R. Bok, I. Park, G. Reed, L. Carvajal, E.J. Small, P. Munster, V.K. Weinberg, J.H. Ardenkjaer-Larsen, A.P. Chen, R.E. Hurd, L.I. Odegardstuen, F.J. Robb, J. Tropp, and J.A. Murray, Metabolic Imaging of Patients with Prostate Cancer Using Hyperpolarized [1-C13]Pyruvate, Sci. Transl. Med. 5 (2013) 198ra108.

[5] Hartmann, S.R. and E.L. Hahn, Nuclear Double Resonance in Rotating Frame, Phys. Rev. 128 (1962) 2042.

[6] Pines, A., M.G. Gibby, and J.S. Waugh, Proton-Enhanced Nmr of Dilute Spins in Solids, J. Chem. Phys. 59 (1973) 569-590.

[7] Pines, A., J.S. Waugh, and M.G. Gibby, Proton-Enhanced Nuclear Induction Spectroscopy Method for High-Resolution NMR of Dilute Spins in Solids, J. Chem. Phys. 56 (1972) 1776.

[8] Lowe, I.J. and D. Tse, Nuclear Spin-Lattice Relaxation Via Paramagnetic Centers, Physical Review 166 (1968) 279.

[9] Tse, D. and S.R. Hartmann, Nuclear Spin-Lattice Relaxation Via Paramagnetic Centers without Spin Diffusion, Phys. Rev. Lett. 21 (1968) 511.

[10] Wenckebach, T., Essentials of Dynamic Nuclear Polarization. 2016: Spindrift Publications; 1st edition (12 May 2016).

[11] Bornet, A., J. Milani, B. Vuichoud, A.J.P. Linde, G. Bodenhausen, and S. Jannin, Microwave frequency modulation to enhance Dissolution Dynamic Nuclear Polarization, Chem. Phys. Lett.602 (2014) 63-67.

[12] Blumberg, W.E., Nuclear Spin-Lattice Relaxation Caused by Paramagnetic Impurities, Phys. Rev. 119 (1960) 79-84.

[13] Smith, A.A., B. Corzilius, A.B. Barnes, T. Maly, and R.G. Griffin, Solid effect dynamic nuclear polarization and polarization pathways, J. Chem. Phys. 136 (2012)

[14] Bornet, A., R. Melzi, S. Jannin, and G. Bodenhausen, Cross Polarization for Dissolution Dynamic Nuclear Polarization Experiments at Readily Accessible Temperatures $1.2<\mathrm{T}<4.2 \mathrm{~K}$, Appl. Magn. Reson. 43 (2012) 107-117.

[15] Bornet, A., R. Melzi, A.J. Perez-Linde, P. Hautle, B. van den Brandt, S. Jannin, and G. Bodenhausen, Boosting Dissolution Dynamic Nuclear Polarization by Cross Polarization, J. Phy. Chem. Lett. 4 (2013) 111-114. 
[Type here]

[16] Jannin, S., A. Bornet, S. Colombo, and G. Bodenhausen, Low-temperature cross polarization in view of enhancing dissolution Dynamic Nuclear Polarization in NMR, Chem. Phys. Lett. 517 (2011) 234-236.

[17] Jannin, S., A. Bornet, R. Melzi, and G. Bodenhausen, High field dynamic nuclear polarization at 6.7 T: Carbon-13 polarization above 70\% within 20 min, Chem. Phys. Lett. 549 (2012) 99-102.

[18] Vuichoud, B., J. Milani, A. Bornet, R. Melzi, S. Jannin, and G. Bodenhausen, Hyperpolarization of Deuterated Metabolites via Remote Cross-Polarization and Dissolution Dynamic Nuclear Polarization, J. Phys. Chem. B 118 (2014) 1411-1415. 